\title{
ARMONIZACIÓN CURRICULAR EN LA EDUCACIÓN SUPERIOR
}

\author{
Dra. Alicia Díaz Alvarado ${ }^{1}$
}

\section{Resumen}

Este artículo aborda la temática de la armonización curricular internacional y explica cómo las características del mundo de hoy han hecho que se convierta en un tema de gran relevancia. Se describen aquí, comparativamente, los logros alcanzados en Europa y América Central en este ámbito y se señalan las dificultades que enfrentan las universidades centroamericanas para mayores avances. Además, se enuncian las acciones que habría que realizar para garantizar condiciones de éxito en la armonización curricular intemacional en la Educación Superior.

\section{Abstract}

This paper approaches the issue of the international curricular harmonization and explains how distinctiveness of today's world makes this a relevant topic. In a comparative analysis, Europe and CentralAmerica achievements in this area are discussed, as well as difficulties that Central American universities face in order to obtain a greater progress. Additionally, the actions that should be taken to guarantee successful conditions in the intemational curricular harmonization in the higher education are presented.

Palabras claves: Currículum - Educación Superior - Integración - Armonización.

\section{Introducción}

a armonización curricular consiste en establecer una cierta convergencia entre diferentes currícula. Este esfuerzo puede circunscribirse a un país o a una región, puede abarcar la totalidad del sistema educativo, o únicamente una

\footnotetext{
'La autora es Máster en Estudios Interdisciplinarios en Psicología y Educación de la Universidad Estatal de California y tiene un doctorado en Educación. Como parte de su trabajo en la Universidad Nacional ha ocupado puestos como la Presidencia del Consejo Central de Posgrado, la Dirección de Docencia y la Presidencia de la Junta de Becas. Ha publicado artículos de investigación sobre la temática de consumo de drogas, tanto en publicaciones periódicas nacionales como internacionales.
} 
parte de éste, como en el caso de que sólo trate de la Educación Superior, y puede cubrir una gran cantidad de disciplinas y áreas del saber o sólo algunas de ellas. Lo central es, entonces, la convergencia, que en cierta medida se ha llamado también homogeneización curricular, integración curricular y, algunas veces, compatibilidad curricular internacional.

Diversos aspectos educacionales pueden ser objeto de armonización: armonización en los títulos que se otorgan, en la duración de los estudios, en los contenidos mínimos, en los niveles de profundización en cada nivel de estudios, en la unidad de medida del esfuerzo que requiere el aprendizaje (crédito universitario), en las formas de expresar el rendimiento de los estudiantes, en el perfil de salida del graduado universitario, en los atestados académicos de los profesores, en la garantía de calidad de los programas, entre otros.

Aunque el tema de la armonización curricular se plantea en este artículo desde una perspectiva internacional, como un fenómeno entre países, también se puede ubicar a nivel de un determinado país. Es decir, aún al interior de un país ocurre que hay aspectos curriculares que no se encuentren armonizados, lo que causa inconvenientes al sistema y afecta a estudiantes, profesores, administradores, y a otros participantes del sistema.

Un ejemplo exitoso de armonización curricular universitaria en nuestro país, lo constituyen los llamados "Estudios Generales", contemplados en el currículum de tres de las cuatro universidades estatales costarricenses. Mediante un acuerdo entre estas universidades, el bloque de cursos de Estudios Generales cursado en una universidad, se reconoce en las otras dos universidades, independientemente de la institución que los impartió, la modalidad metodológica, las temáticas estudiadas y cualquier otro factor en que se puedan establecer variaciones.

Igualmente, se han armonizado en las universidades costarricenses, entre otros, el concepto de crédito, la nomenclatura de los diversos niveles de estudios y la duración de éstos y algunos aspectos de los estudios de posgrado. Sin embargo quedan aún muchos temas por armonizar y en los cuales debe mantenerse un esfuerzo persistente para alcanzar mayores niveles de integración univèrsitaria.

Este trabajo pretende incursionar en el tema de la armonización curricular en la educación superior a nivel de diferentes países, con la intención de promover una mayor apertura de los universitarios hacia la armonización, mediante el conocimiento de su relevancia actual y de su relación con otros fenómenos contemporáneos. 


\section{Contextualización e implicaciones para la educación superior}

El fenómeno de la armonización curricular debe ser analizado a la luz del contexto actual, para visualizar su significado e importancia de acuerdo con las condiciones nacionales e internacionales vigentes, entre las cuales destacan las siguientes:

\subsection{Avances tecnológicos actuales}

Los avances tecnológicos actuales han provocado un acercamiento entre los pueblos. La distancia, en términos geográficos, ha dejado de ser un factor de lejanía, por los recursos disponibles en telecomunicaciones. Así por ejemplo, el teléfono, el fax, las videograbadoras, la televisión, la transmisión vía satélite, y otros recursos similares, contribuyen con esta reducción de las distancias. La fabricación de sofisticados aviones y la construcción de autopistas que permiten viajar a altas velocidades, ayudan en esta reducción de distancias. Igualmente ocurre con las fronteras, pues tienen en la actualidad un significado completamente diferente al que tenían hace unos años, dado que no se requiere cruzarlas para entrar en contacto con otros pueblos. Además, hay que considerar que esta época se caracteriza por la simultaneidad: no hay que esperar ni días ni horas para conocer una noticia.

La información es simultánea y las cadenas de reacciones a estas noticias también lo son. Esto ha revolucionado el mundo, en comparación con el mundo que vivieron las generaciones pasadas.

Como toda institución y fenómeno social, la educación superior se ve influida por estos adelantos tecnológicos. La ciencia no tiene fronteras. Los académicos y estudiantes deben comunicarse entre sí para conocer y participar del desarrollo científico en el momento oportuno. Las universidades deben promover estas comunicaciones y contactos científicos, en nombre de la actualización que requiere su personal y sus estudiantes y en nombre de la difusión del conocimiento. El intercambio internacional debe ser altamente valorado y promovido al interior de las universidades, pues de éste depende, en gran medida, el desarrollo académico universitario.

En nuestro país, las universidades han tomado conciencia de esta situación y por ello han hecho importantes inversiones para incorporar tecnología en los procesos académicos. En la actualidad, la gran mayoría de los universitarios tiene una cuenta electrónica y acceso a equipo computacional, con lo cual puede ser parte de redes de información, acceso a bibliotecas electrónicas, 
páginas electrónicas de universidades y de asociaciones profesionales, lo que da la oportunidad de establecer comunicaciones con colegas en otras partes del mundo, y obtener la información actualizada que requiere para realizar su trabajo.

Las telecomunicaciones y sus aportes en el plano académico contribuyen con la armonización curricular, pues facilitan el conocimiento de las diversas formas en que se plantea el currículum en diferentes lugares, y con ello permiten la comprensión, valoración, comparación y análisis de los programas, lo cual enriquece el pensamiento científico.

\subsection{Era de la mundialización}

La mundialización es un fenómeno que sugiere una tendencia que envuelve a todo el planeta. Lleva implícita la idea de unificar y de asumir los diferentes procesos a escala mundial. Así por ejemplo, en estos tiempos lo relevante ya no es tanto la economía nacional como la economía mundial y lo mismo ocurre con el comercio, la producción industrial, las finanzas, la política, etc. Esta mundialización ha cambiado todo el patrón de las relaciones internacionales.

Desde el punto de vista de la educación superior, también se han generado cambios en las relaciones entre las instituciones, y a partir de ellos se han identificado nuevos retos y nuevas demandas. Uno de estos retos es la promoción de la cooperación internacional y el intercambio académico entre las universidades. Entre las consideraciones que dieron lugar a este reto, está la confianza implícita de que las comunidades académicas son capaces de expresar, mantener, promover y replantear acuerdos mutuos entre las naciones, aún cuando esto sea imposible por otros canales. Esto significa que las universidades tienen un papel que cumplir en los procesos de integración internacional (Cummings, 2001).

A partir de lo anterior, se visualiza otro reto para las universidades: plantear, internamente, los cambios estructurales, conceptuales y operativos que se requieran para poder cumplir con estas nuevas expectativas de la educación superior. Obviamente, es muy difícil cumplir con estas expectativas, si se tienen características de la universidad tradicional, con esquemas académicos muy rígidos y personal formado en una época completamente diferente.

Otro reto importante es desarrollar formas de financiamiento que permitan a estas instituciones cumplir con los programas internacionales esperados y, simultáneamente, mantener su autonomía y tener la libertad para poner en marcha diversas iniciativas. Es claro también que el financiamiento tradicional es insuficiente. 


\subsection{Esfuerzos de integración económica de diversos países}

Diversos países y regiones están haciendo esfuerzos por la integración como una estrategia para fortalecerse, racionalizar los recursos, disfrutar del enriquecimiento que ocurre cuando hay apertura a otros pueblos, propiciar el acceso, la diversificación y la divulgación de productos, servicios y otros bienes y mejorar las ganancias derivadas del comercio internacional. Uno de los modelos lo constituye la Comunidad Europea, cuya integración es muy visible en su moneda, en las facilidades para el desplazamiento de un país a otro, en la apertura para el comercio de bienes y servicios al interior de la región y en sus avances hacia la integración política.

También los tratados de libre comercio son manifestaciones de integración económica, la cual, inevitablemente, lleva aspectos que van más allá de lo económico, pues toca, al menos, lo político, lo académico, lo social y lo cultural. Si en el entorno hay integración, y si la Educación Superior debe responder a las necesidades del entorno, entonces, la Educación Superior debe, también, asumir elementos de integración.

Las universidades no pueden quedarse al margen de los esfuerzos de integración de ciertos bloques de países. Deben estar presentes para dar su aporte crítico, analizar y dar a conocer su punto de vista, respaldar los elementos valiosos, rechazar los que se consideran inapropiados, visualizar efectos a corto, mediano y largo plazo, promover medidas que contrarresten las consecuencias negativas o que apoyen aquellas que son positivas, entre otras.

Para que este aporte de las universidades sea posible, es necesario contar con cierta armonización curricular. Las universidades deben coincidir en poner el tema de la integración económica en sus agendas y aquellas que han avanzado más, pueden contribuir con las que han avanzado menos y deben establecer comunicaciones cercanas para compartir los conocimientos. Esto sólo es posible si tienen un común denominador que le dé sentido a los esfuerzos compartidos. La armonización curricular puede ser punto de partida y, a la vez, consecuencia del interés común por hacer frente, de la mejor manera, a la integración económica.

\subsection{Fenómenos migratorios}

La migración es un fenómeno que se ve favorecido en la actualidad por diversos factores, entre ellos, por las facilidades de comunicación y de traslado y por el acercamiento entre los pueblos. Esto también impacta la Educación 
Superior, en la medida en que debe garantizar cierta convergencia curricular para que sea posible la continuidad de los estudios en los casos de migrantes; facilitar la inserción de unos estudios en otros (como en el caso de intercambio estudiantil) y aprovechar plenamente el recurso humano profesional que se desplaza de un país a otro. Todo esto requiere conocer la equivalencia de los estudios realizados en el país de origen, en relación con los que se ofrecen en el país de llegada. Además, en condiciones de competencia entre instituciones, es más atractiva una oferta curricular que permita armonizar con estándares internacionales que aquella que sólo tiene un referente local.

\subsection{Internacionalización de las universidades}

En la era de la internacionalización de las universidades cobran gran importancia todas las formas de colaboración interuniversitaria en las que participen instituciones de varios países. La colaboración mutua se puede orientar al desarrollo de planes de estudio o de proyectos de investigación o acción social. Algunos ejemplos son los siguientes:

- Carreras compartidas y titulación compartida

- Investigaciones compartidas

- Cooperación interuniversitaria en servicios bibliotecarios.

- Pasantías para profesores e investigadores y estudiantes

- Intercambio de aprendizajes, de innovaciones, de procedimientos y divulgación internacional de la producción (seminarios y talleres)

- $\quad$ Procesos de acreditación (estándares internacionales de calidad)

- Flexibilidad curricular (valor otorgado a estudios en otros países).

- $\quad$ Financiamiento de procesos de unas universidades a otras.

- Apertura para la admisión y becas para estudiantes extranjeros

- $\quad$ Programas virtuales

En todos los casos la integración académica tiene, como condición previa, una cierta convergencia académica, a efecto de utilizar un mismo lenguaje, propiciar un mejor entendimiento entre los académicos y estudiantes participantes, facilitar la inserción y la continuidad de los estudios mediante el desarrollo de programas en forma combinada, todo lo cual refuerza que las universidades deben promover la cultura de la integración universitaria, y en su campo específico, la cultura de la armonización académica. 


\section{Experiencias en armonización curricular en Educación Superior}

En el ámbito internacional, se han realizado diversos esfuerzos dirigidos a la armonización curricular universitaria. Estos se han visto limitados por los desarrollos desiguales de los países, los cuales generan, a la vez, grandes desigualdades en el desarrollo educativo, social, económico y tecnológico.

Una condición de mayor apertura significaría un mejor aprovechamiento de las oportunidades, participación en redes temáticas y profesionales que le abrirían puertas a las instituciones; significaría un mayor impacto nacional $\mathrm{y}$, por ende, el respectivo apoyo de las instancias beneficiadas, y en general la consolidación de una serie de condiciones favorables al desarrollo institucional.

Las experiencias de armonización curricular que se han realizado internacionalmente son de naturaleza variada. Algunas se ubican en áreas disciplinarias particulares, por ejemplo en América del Sur se han realizado esfuerzos exitosos en las áreas de Farmacia y Bibliotecología, con participación de diferentes países de la región.

Otras experiencias se han planteado con una cobertura académica más amplia, pues no se circunscriben a una disciplina. Este es el caso de los logros alcanzados en la Comunidad Europea, los cuales contemplan acuerdos generales entre los sistemas universitarios los países participantes, lo que les permite una gran diversidad y flexibilidad de opciones, como la movilidad docente y estudiantil, que se está llevando a cabo con una gran aceptación.

En forma paralela y con miras a la armonización curricular, los sistemas universitarios europeos han hecho cambios internos, tratando de hacer sus programas de estudio más compatibles con aquellos realizados en otros países. Este esfuerzo se enmarca en las políticas institucionales de desarrollo de las universidades y en la política exterior de cada nación, coordinación que fortalece el impacto de estos esfuerzos y aumenta sus posibilidades de éxito

A continuación, se presentan dos experiencias de armonización curricular: una es la que se ha puesto en práctica en Alemania, que refleja el pensamiento europeo, y la otra es la que se está intentando en la región de Centroamérica a través de la Confederación Superior Universitaria Centroamericana (CSUCA), la cual es, igualmente, un buen reflejo del pensamiento centroamericano y de las condiciones prevalecientes de la región.

\subsection{Acciones realizadas en Alemania para la armonización curricular}

Las características del contexto actual y los acontecimientos que se desarrollaron en el continente europeo en el siglo XX (guerras mundiales, 
destrucción, separación entre países y posterior reunificación de esos países, entre otros) provocaron cambios significativos en el patrón de relaciones exteriores de los gobiernos y, por ende, en el patrón que asumieron las universidades al interactuar con otras universidades. Estos procesos se dieron particularmente en la República Federal de Alemania, por ser esta una de las protagonistas principales en los acontecimientos mencionados.

Entre los cambios que se produjeron en las universidades alemanas estuvieron la coordinación y la promoción de las relaciones internacionales, como un medio para alcanzar su propio desarrollo institucional. Consistentemente, tomó fuerza la movilidad estudiantil, tanto para atraer estudiantes extranjeros que cursaran estudios en las universidades alemanas, como para promover que los estudiantes alemanes realizaran estudios en el extranjero.

Algunas de las acciones de armonización curricular que se desarrollaron en el sistema universitario alemán en la última década del siglo XX, fueron:

- $\quad$ Apertura de programas de posgrado especiales para estudiantes de países en vías de desarrollo, en concordancia con las necesidades e intereses de estos países. Estos posgrados se organizan independientemente de los programas regulares de la universidad alemana correspondiente, para ofrecer a los estudiantes extranjeros, facilidades para aprender el idioma alemán e insertarse en la universidad sin haber recibido, en Alemania, la formación de base que requieren los estudios cursados (Hockschul Rectoren Konferenz-HRK-, 1998²).

- Mayor flexibilidad al valorar la certificación de estudios secundarios aportada por extranjeros que solicitan admisión a las universidades alemanas.

- $\quad$ Apertura de una gama de oportunidades para el aprendizaje del idioma alemán y de la cultura alemana.

- $\quad$ Apoyo a los estudiantes extranjeros para el cumplimiento de los requisitos legales establecidos para su estadía en Alemania: obtención de visa, seguro de salud y permisos de trabajo. El apoyo también cubre facilidades para su hospedaje y para la instalación de familiares, cuando fuera necesario.

- $\quad$ Puesta en marcha de un nuevo esquema de titulación de los estudios realizados en Educación Superior, en Alemania, con la finalidad de lograr mayor compatibilidad internacional. La estrategia en este caso fue dar más flexibilidad a los estudiantes en cuanto a la programación de

\footnotetext{
2HRK indica Hockschul Rectoren Konferenz. institución que agrupa a los rectores de las universidades alemanas y en la cual se toman las decisiones sobre las políticas del sistema universitario alemán.
} 
sus estudios y permitir el otorgamiento de títulos intermedios que anteriormente no existían. El sistema alemán contemplaba una forma de titulación muy compleja, con una amplia diversidad de sistemas para la obtención del título y una sola línea curricular desde el ingreso a la Universidad hasta alcanzar un título equivalente a Maestría, con una duración aproximada de 4 ó 5 años, lo que daba al estudiante los créditos académicos para ingresar a un Doctorado. Actualmente, como parte de los esfuerzos de armonización curricular, también existe la opción de obtener un título por un primer nivel de estudios equivalente a un $\mathrm{Ba}$ chillerato, cuya duración es de tres o cuatro años y luego, con uno o dos años más de estudios, obtener un título equivalente a una Maestría, lo que también permite el acceso a un Doctorado. En esta modalidad que tiene un primer y un segundo título, en algunos casos se otorga la admisión a un Doctorado a partir del primer título. Como se puede apreciar, este es un cambio de gran envergadura en el sistema universitario alemán y es, por lo tanto, una muestra de la voluntad por flexibilizar y por armonizar e internacionalizar el currículum.

- La creatividad universitaria y las fuertes tendencias hacia la internacionalización han dado lugar a otras formas de armonización curricular en las universidades, particularmente pensadas en términos de la cooperación con los países del tercer mundo. Ejemplo de esto son los "programas sandwich", en los cuales un estudiante cumple una parte de sus estudios en un país extranjero y la otra en su país de origen. Otro ejemplo lo constituyen los programas especiales de posgrado, en los que el estudiante obtiene una formación que lo prepara para ciertas tareas específicas o para el trabajo con determinadas poblaciones. En estos casos, la Universidad puede ofrecer un posgrado en "tópicos especiales", el cual abre la oportunidad para que los estudiantes extranjeros definan el tópico de su interés, según las necesidades de su país de origen.

A manera de acompañamiento de estas acciones, las universidades han promovido esfuerzos de divulgación para asegurar una información correcta y oportuna para los estudiantes extranjeros: capacitaron a los funcionarios de embajadas, consulados, institutos y otras instituciones alemanas en el extranjero, de manera que estuvieran capacitados para orientar, integralmente, a los interesados en estudiar en Alemania; incrementaron su participación en ferias educacionales internacionales e incorporaron información de procesos de capacitación universitaria en la documentación emitida por la Comunidad 
Europea, promoviendo así que ésta llegara no sólo a las universidades extranjeras, sino también a organizaciones profesionales, comerciales y productivas.

Otra forma de acompañamiento consistió en mantener cercanía con los países y con las universidades con las que se pretende la armonización, por lo cual, parte del esfuerzo consistió en buscar fondos que permitieran las visitas mutuas de los profesores de las universidades cooperantes y contar con espacios en los que se planearan y coordinaran eventos académicos como cursos, seminarios, conferencias, investigaciones, entre otros.

Como se nota en el ejemplo anterior, la armonización curricular ha cobrado tales dimensiones en la actualidad que ha motivado iniciativas sumamente novedosas y revolucionarias en aquellos países que han visualizado su relevancia. Además, las acciones particulares que se han derivado de la armonización curricular han sido tan importantes en Europa que incluso se puede afirmar que cumplieron un papel fundamental en la integración europea al facilitar los contactos personales y profesionales, al estrechar los lazos entre las comunidades académicas y al reducir el impacto de prejuicios, resentimientos y relaciones de competencia entre los pueblos.

\subsection{Acciones orientadas a la armonización curricular en América Central}

A nivel de la región centroamericana, es posible identificar esfuerzos de armonización curricular. En esto ha jugado un papel muy importante la Confederación Superior Universitaria Centroamericana-CSUCA ${ }^{3}$, que integra a las universidades estatales centroamericanas. Su misma existencia constituye, de por sí, un acercamiento entre los sistemas de educación superior en la región, con miras a la coordinación y colaboración, para estimular el crecimiento y la consolidación de las universidades que conforman el sistema.

El CSUCA ha concentrado su ámbito de acción en la integración académica, a pesar de que en el ambiente han estado presentes, desde su creación en 1948, otros esfuerzos de integración centroamericana, de carácter económico y político, que no han sido exitosos. Las dificultades de la integración centroamericana han tenido un impacto en el mismo CSUCA. Por ello, este organismo ha estado muy debilitado en algunos períodos de su historia, sin embargo, ha sido capaz de sobrevivir y mantenerse, a pesar de los diferentes conflictos en la región.

\footnotetext{
$\overline{3}$ CSUCA - Confederación Superior Universitaria Centroamericana, es la instancia que reúne a los rectores y representantes estudiantiles de las universidades estatales centroamericanas y en ella se coordina y se toman acuerdos que orientan el accionar de las universidadesestatales de la región.
} 
Entre los logros que se pueden atribuir a la coordinación que promueve el CSUCA están: la aprobación de acuerdos para la armonización y colaboración entre las instituciones de educación superior centroamericana, la creación de redes y sistemas regionales en torno a ámbitos específicos y el intercambio académico con universidades del resto del mundo, actuando como bloque de universidades y de países.

Los acuerdos que contempla la constitución del CSUCA aluden al desarrollo de la educación superior en la región, lo que ha dado lugar a que se generen acciones para una comunicación efectiva entre las universidades. Esto incluye acciones para definir términos como crédito universitario, grado y posgrado y sus diferentes niveles. Muchas discusiones, propuestas y encuentros tuvieron lugar en este proceso, lo que sugiere que, a pesar de que la región es relativamente pequeña en términos de su área geográfica, es grande en su diversidad y heterogeneidad, factor que incide en los logros alcanzados por la armonización curricular.

En cuanto a la creación de redes y sistemas regionales, hay que señalar que el CSUCA ha sido protagonista en los esfuerzos de integración de la región. Entre estos esfuerzos se pueden mencionar: la Red de Sistemas de Información Documental - RED SIID, el Sistema de Carreras y Posgrados Regionales -SICAR, el Sistema Centroamericano de Evaluación y Acreditación de la Educación Superior- SICEVAES, el Consejo Regional de Vida Estudiantil -CONREVE y el Programa de Enseñanza de los Derechos Humanos en la Educación Superior.

Algunos de estos programas han tenido una amplia divulgación en las universidades participantes, aunque en otras, el impacto ha sido menor; sin 
embargo, como bloque se ha logrado dar un impulso a la temática específica que se aborda. Debe destacarse que estos procesos constituyen, en sí mismos, un aporte considerable dado que permiten ir construyendo una plataforma colectiva, centroamericana, de conocimiento y colaboración, lo cual finalmente podría tener consecuencias favorables para la armonización curricular.

Cabe mencionar que cada uno de estos sistemas establece acciones específicas que también aportan al proceso de integración académica: crean carreras con temáticas centroamericanas, propician encuentros de académicos y estudiantes, comparten temáticas de interés en la región, analizan situaciones y desarrollan proyectos de cobertura centroamericana, se ejecutan experiencias innovadoras, se crean bases de datos con información de las universidades, se plantean acuerdos que deben asumir las universidades, entre otras acciones. Todo esto permite ir construyendo las bases que preparan para alcanzar mayores niveles de integración en el futuro.

En el ámbito de las relaciones con países que están fuera de la región centroamericana, el CSUCA ha tenido un papel muy importante al presentarse al resto del mundo como un espacio en el que los países centroamericanos se encuentran integrados. Cabe recordar que algunos países sólo apoyan esfuerzos en los que haya un impacto significativo para una región amplia y no para un espacio local reducido. Esta política ha dejado por fuera el financiamiento de iniciativas de una única universidad, en la que se plantean proyectos aislados que benefician sólo a un determinado país (CSUCA, 2001). El CSUCA ha servido para que las universidades centroamericanas aprovechen, como bloque, las oportunidades de colaboración, financiamiento e intercambio académico con universidades del resto del mundo, creando así otra plataforma de acercamiento que favorece la armonización curricular.

Los resultados de estos esfuerzos han sido de gran importancia en la región centroamericana, pues han ayudado a la construcción de vínculos que, desde su concepción, son imprescindibles para lograr resultados, con lo cual se abona a la creación de una mentalidad orientada a la integración centroamericana. Las universidades contraparte también juegan un papel determinante, pues su visión y su manera de relacionarse con cada persona que participa de estos proyectos. revela la concepción de un ciudadano centroamericano y deja de lado su identidad nacional, forzando así a estos participantes a identificarse con los intereses de la región, y orienta sus acciones a la defensa y promoción de estos intereses comunes. En el mediano plazo, es de esperar que el efecto de estos esfuerzos también abone a los procesos de armonización curricular en Centroamérica. 


\section{Condiciones de logro y acciones hacia la armonización curricular}

El éxito en los esfuerzos de armonización curricular requiere de condiciones que son imprescindibles para que esta sea una realidad. No basta, entonces, con la voluntad de armonizar el currículum, también hay que plantear la necesidad de condiciones mínimas que preparen para esta armonización y para el cambio de mentalidad que ésta implica.

Entre las condiciones más importantes, se encuentra una cierta homologación de los niveles de calidad y desarrollo en las universidades. Este factor merece atención en el área centroamericana, dado el desarrollo desigual de las universidades. La desigualdad se puede percibir en oferta académica, capacidad investigativa, formación del recurso humano, productividad, financiamiento y cobertura estudiantil, entre otros. Ante esto, cabe destacar la necesidad de dar un mayor impulso a las acciones colaborativas al interior de la región, lo que permite equilibrar y ayudar a las instituciones a superar sus deficiencias. Esta colaboración implica solidaridad y una identidad centroamericanista que la región debe fortalecer.

Conocer los datos educativos relevantes de los estados participantes implica contar con información ordenada, sistematizada, válida, confiable, exhaustiva, pertinente y oportuna sobre los programas y sus características; esta es otra condición para la armonización, misma que es una de las limitaciones más importantes que han enfrentado quienes han hecho esfuerzos por la armonización curricular en las universidades centroamericanas y latinoamericanas. La carencia de información impide que se puedan determinar las pautas concretas que orienten las acciones conjuntas entre las universidades participantes.

Además, viabilizar la armonización curricular implica definir y poner en marcha mecanismos jurídicos, administrativos y académicos que posibiliten una verdadera compatibilización en los estudios. Las universidades centroamericanas deben organizarse para discutir este tema y poner en marcha procesos que, efectivamente, propicien la armonización curricular.

El avance hacia la armonización curricular requiere de innovaciones que hagan posible esta armonización. Al respecto, hay que buscar formas de plantear el currículum con miras a la compatibilización. En algunos casos, se ha utilizado el currículum por competencias como un medio eficaz para la armonización, lo que implica armonizar en términos de las competencias desarrolladas en el proceso de estudios de una carrera. Por ejemplo, en la compatibilización de programas de estudio de Bibliotecología que se ha trabajado 
en el MERCOSUR, se ha planteado, como herramienta básica, la definición de las competencias que debe poseer un profesional en Bibliotecología de la región. Por otro lado, también hay que explorar cuáles cambios se requieren en cuanto a enfoques curriculares y estrategias pedagógicas, para impulsar la integración académica. Este punto ha sido polémico en Centroamérica, por lo que se necesita una discusión profunda, a la luz de las nuevas tendencias mundiales, para avanzar hacia la armonización curricular.

Otro aspecto importante es poner en ejecución programas de recepción de estudiantes internacionales. Esto permite prepararse para enfrentar la diversidad internacional, a la vez que promueve apertura hacia este tipo de experiencias: ayuda a identificar la riqueza de esta diversidad, contribuye con la eliminación de los prejuicios que muchas veces obstaculizan los programas, facilita que se aproveche la iniciativa y la creatividad para generar ideas nuevas y, en general, construir condiciones para su fortalecimiento. Cabe mencionar que, además de las condiciones académicas, es necesario también desarrollar condiciones financieras, sociales y legales, de manera que los estudiantes extranjeros tengan confianza en la seguridad, acompañamiento y apoyo que ofrece la universidad anfitriona, no sólo para todo lo que requiere dentro del campus universitario, sino también para cumplir con los requisitos de visa y otras regulaciones nacionales para los extranjeros.

En la movilidad académica, debe coordinarse cercanamente con los organismos gubernamentales que regulan la visita y permanencia de extranjeros. A partir de esta coordinación, la universidad tiene que tomar medidas para asegurar que los universitarios visitantes estén a derecho con las leyes y, simultáneamente, que la documentación solicitada por la institución sea viable y relevante.

Otra condición fundamental para la armonización curricular es la flexibilidad en los procesos de reconocimiento y la equiparación de estudios. La evaluación de los atestados de un estudiante requiere un replanteamiento y nuevos esquemas, en los que se respete y se valore el esfuerzo realizado por las instituciones de educación superior de las diversas naciones y se pongan en práctica mecanismos que permitan equilibrar estudios, más que rechazar un estudiante por una baja valoración de sus estudios previos y por la identificación de deficiencias en su formación. En este caso, los estudios de nivelación cumplen un papel fundamental, sobretodo cuando la movilidad estudiantil se realiza desde países con niveles inferiores de desarrollo hacia países más desarrollados.

La capacitación de las comunidades universitarias para aceptar, propiciar, participar y favorecer la armonización curricular es un aspecto esencial. 
Los diversos actores de estos procesos, docentes y estudiantes, deben estar convencidos de que la convergencia curricular es importante y han de contar con argumentos válidos para ello. Por esto, se convierte en necesidad asegurar este convencimiento y apoyo actitudinal, para lo cual se pueden utilizar sesiones de capacitación, pasantías y participación en las modalidades de acción académica que plantean los procesos de internacionalización de las universidades.

Por último, una condición más para el éxito de los esfuerzos de armonización curricular radica en el dominio de lenguas extranjeras por parte de la comunidad académica, situación que es bastante débil en el área centroamericana. Es importante promover pasantías, cursos de idioma, estudios en el extranjero y otras medidas similares, con el objetivo de incentivar que los profesores y estudiantes dominen al menos una lengua extranjera. Estas experiencias internacionales tienen una ventaja secundaria que consiste en presenciar la manera en que se realizan los procesos universitarios en otras latitudes, y con ello flexibilizar el pensamiento, las actitudes y las emociones. El aprendizaje de un idioma extranjero y el cambio personal asociado a una experiencia internacional serán, indudablemente, portadores de una visión prospectiva de mayor apertura para las universidades. Sólo así será posible la comunicación y la comprensión necesaria para llevar a cabo, a plenitud, los procesos de armonización curricular internacional y con ello abrir nuevas puertas para el desarrollo de las universidades de la región. 


\section{REFERENCIAS}

Consejo Superior Universitario Centroamericano - CSUCA (2001). Centroamérica: Universidad pública e integración de la educación superior. Serie "Educación Superior en Centroamérica".

Cummings, W. (2001). Current Challenges in International Education. ERIC DIGEST ED464523.

Hochschul Rectoren Konferenz (HRK) (1998). Academic equivalences and recognition: Bilateral agreements of the HRK and Documents 1990-1998. Vol. 1. Bonn. 